\title{
Modified Newtonian Dynamics as an Entropic Force
}

\author{
Diego A. Carranza, Sergio Mendoza \\ Instituto de Astronoma, Universidad Nacional Autónoma de México, México D.F., México \\ Email: dortiz@astro.unam.mx, sergio@astro.unam.mx
}

Received 15 February 2015; accepted 22 May 2015; published 25 May 2015

Copyright (C) 2015 by authors and Scientific Research Publishing Inc.

This work is licensed under the Creative Commons Attribution International License (CC BY).

http://creativecommons.org/licenses/by/4.0/

(c) (i) Open Access

\begin{abstract}
Under natural assumptions on the thermodynamic properties of space and time with the holographic principle, we reproduce a MOND-like behaviour of gravity on particular scales of mass and length, where Newtonian gravity requires a modification or extension if no dark matter component is introduced in the description of gravitational phenomena. The result is directly obtained with the assumption that a fundamental constant of nature with dimensions of acceleration needs to be introduced into gravitational interactions. This in turn allows for modifications or extensions of the equipartion law and/or the holographic principle. In other words, MOND-like phenomenology can be reproduced when appropriate generalised concepts at the thermodynamical level of space and/or at the holographic principle are introduced. Thermodynamical modifications are reflected in extensions to the equipartition law which occur when the temperature of the system drops below a critical value, equals to Unruh's temperature evaluated at the acceleration constant scale introduced for the description of the gravitational phenomena. Our calculations extend the ones by [1] in which Newtonian gravity is shown to be an emergent phenomenon, and together with it reinforces the idea that gravity at all scales is emergent.
\end{abstract}

\section{Keywords}

Alternative Theories of Gravity, Modified Newtonian Dynamics, Classical Black Holes, Entropy, Thermodynamics of Black Holes

\section{Introduction}

The laws for black hole mechanics have suggested a remarkable similarity with the three laws of thermodynamics, in such a way that quantities associated to black hole properties have their corresponding thermodynamic equivalent interpretation [2]-[4]. In particular, the black hole area-which is determined by its horizon-is related to the associated black hole entropy, in the sense that it cannot decrease in time under any physical process 
on a closed system. The temperature of the black hole is given by the Hawking-Zeldovich temperature and is inversely proportional to the mass of the black hole [5] [6]. The well known interpretation of entropy as a quantity that offers a measure of non-available information—or disorder—in a system, has leaded directly to the idea that the increase in entropy, and therefore in area, is due to the loss of information. This is due to the fact that when a particle crosses the horizon it has no more causal relation with the rest of the universe [2].

All the above suggest the possibility for a deep relation between thermodynamics and gravity. This has been studied mainly in the relativistic regime under the concept of emergent gravity, considering thermodynamics as a more fundamental theory from which, general relativity can be derived (see e.g. [7] and references therein). Using a metric treatment of the thermodynamic variables in a curved space-time, [8] has been able to derive Einstein's field equations. In the non-relativistic regime, [1] used very simple assumptions about space, energy and information in order to show that the first law of thermodynamics, along with an entropy formula, leads directly to Newton's law of gravity.

In recent years, a growing number of independent observations have suggested that gravity requires modification [9]-[14] and not the inclusion of unknown dark matter entities. All these facts are encouraging, since similar arguments like Verlinde's ones can be used to search for a more profound fundamental basis for an extended theory of gravity like the one proposed by [15]. This approach is based on Buckingham's $\Pi$ theorem of dimensional analysis, and deals with the problem of a test particle under a gravitational field generated by a central point mass $M$ in an extended regime of gravity. Such analysis gives the general form for the acceleration $a$ experienced by a test particle at a distance $r$ from the central mass:

$$
a=a_{0} f(x), \quad \text { with } x:=\frac{l_{M}}{r}:=\frac{1}{r}\left(\frac{G M}{a_{0}}\right)^{1 / 2},
$$

where $a_{0}$ is Milgrom's acceleration constant [16], introduced as an extra fundamental constant of nature when extended gravitational phenomena is described. The unknown dimensionless function $f(x)$ depends only on the dimensionless quantity $x$, which according to the $\Pi$ theorem, is the key parameter in a description of extended gravity. The function $f(x)$ is such that: $f(x) \rightarrow x^{2}$ when $x \gg 1$, converging to Newtonian gravity; and $f(x) \rightarrow x$ when $x \ll 1$, reaching the deep MONDian regime of gravity. The authors also showed that the transition function could be written as:

$$
f(x)=x \frac{1 \pm x^{n+1}}{1 \pm x^{n}}
$$

where $n$ is a constant that must be fixed via astronomical observations. A large value of $n$ means that the function $f(x)$ abruptly changes from a MONDian to a Newtonian regime of gravity about $x \approx 1$. Small values of $n$ yield soft transitions about the same point.

For the case of spherically symmetric mass distribution, this extended Newtonian gravity approach proposal reproduces a MOND-like phenomenology [16] and has proven to be in good agreement with observations in astrophysical systems across different scales without invoking any dark matter component [9] [10] [15] [17]-[19].

In another attempt to obtain the MOND-like force formula, [20] have followed Verlinde's work assuming that the equipartition law of energy is modified. In this approach, a Debye's function is introduced, and becomes identified as the MOND interpolation function $\mu\left(a / a_{0}\right)$. The reason of doing so is simply to satisfy Newtonian and MONDian regimes of gravity at their corresponding limits. Furthermore, they also give an expression for the value of Milgrom's acceleration constant in terms of Debye's temperature $T_{\mathrm{D}}$ :

$$
a_{0}=\frac{12 c k_{\mathrm{B}} T_{\mathrm{D}}}{\pi \hbar},
$$

thus interpreting it as a cut off temperature below which, modifications to the equipartition law must occur. This approach takes into account the fact that the dynamical sector needs to be modified and so, the validity of Newton's law of gravitation remains unaffected. As explained by the dimensional analysis of [15], the modification must occur in the gravitational sector and rather than working with a transition function $\mu\left(a / a_{0}\right)$, the extension is carried out through the inclusion of a transition function $f(x)$.

In this work, we show how, using arguments about thermodynamics and information, it is possible to derive 
in several ways an equation for the gravitational force in an extended modified gravity regime, which supports the idea that gravity can be understood as an emergent force, i.e. a consequence of deeper fundamental principles. The article is organised as follows: in Section 2 we review the main hypothesis made by [1], and then we use dimensional analysis arguments to study modifications to the equipartition law of energy via two approaches, one of them purely thermodynamic, and the other only gravitational. Using another point of view, Section 3 is devoted to study possible modifications to the holographic principle, specifically, to the number of bits contained inside a screen under the assumption that Milgrom's acceleration constant $a_{0}$ is a fundamental constant of nature. Finally, in Section 4 we discuss our main results.

\section{Modifications to the Equipartition Law}

We begin this section reviewing briefly some of the main ideas and hypothesis made in Verlinde's work [1]. One of the most important assumptions made by him, is that the information describing a physical system is stored on spatial surfaces, or screens, that are ruled by the holographic principle. Every surface behaves as a "stretched horizon" of a black hole (although in this case it has no physical properties like density or surface pressure), and when a particle interacts with it, the entropy, and consequently, the amount of information gets affected. In principle we do not know the shape of the surfaces, so for simplicity we can consider each screen as closed and spherical with radius $r$. Each surface contains $N$ bits of information. One can also think that on each fundamental Planck square area, the maximum information that can be stored is one bit. This length is constructed with three fundamental constants of nature: 1 ) Newton's constant of gravity $G, 2$ ) the velocity of light $c$ and 3) Planck's constant $\hbar$. With these assumptions, the number of bits $N$ stored on each screen can be expressed as:

$$
N=\frac{A}{l_{\mathrm{P}}^{2}}=\frac{4 \pi r^{2}}{l_{\mathrm{P}}^{2}},
$$

being $A$ the area of the spherical screen and $l_{\mathrm{P}}^{2}=G \hbar / c^{3}$ the Planck area.

The main motivation by [1] to think of gravity as a force related to entropy has its origin on the restitutive force that acts on a polymer when it suffers a displacement $\Delta x$. This force tends to restore the polymer to its original position since this configuration maximises the entropy. The link with gravitation consists on a similar idea, for which there is an entropic force that emerges as a consequence of the system searching for a configuration of maximum entropy when a particle approaches a given screen. We assume that inside the screen the dynamics allow us to define energy, and consequently the associated mass $M$ and temperature $T$ are well defined quantities. With this, we can use the first law of thermodynamics to find the force $F$ associated to changes in the stored information, i.e. due to a change in entropy $\Delta S$ given by:

$$
F \Delta x=T \Delta S \text {, }
$$

for a constant volume. Let us now find the expression for the gravitational force by considering gravity as an entropic force. For this, we follow the approach by [1] [8] analysing the behaviour of a test mass $m$ particle near a black hole horizon. At a distance of one Compton length from the horizon, the particle can be considered to be part of the black hole and so, its entropy is increased in the following way:

$$
\Delta S=2 \pi k_{\mathrm{B}} \frac{m c}{\hbar} \Delta x,
$$

when a displacement $\Delta x$ occurs. In other words, a change in the particle position causes an increment on the system's entropy, such that it tries to maximise it and as such, the horizon can be substituted by a screen. The other key assumption to make is that the energy contained inside the surface satisfies the principle of equipartition, and that it is equal to $M c^{2}$, where $M$ is its associated mass. At this point, we take an approach similar to the one followed by [20], i.e. we search for a modification of the equipartition law. This can be achieved with the help of Buckingham's theorem of dimensional analysis, since it provides a way to find the general form for the energy when modifying effects are considered.

To do so, note that the dimensional relevant quantities of the problem are the energy $E$ associated to the screen, it's temperature $T$-or more important for a dimensional analysis treatment its energetic temperature $k_{\mathrm{B}} T$ (where $k_{\mathrm{B}}$ is Boltzmann's constant), the speed of light $c$ and Planck's constant $\hbar$. Since we are study- 
ing a gravitational problem in an extended regime, Milgrom's constant $a_{0}$ is also introduced, along with Newton's constant of gravity $G$. The associated mass $M$ of the spherical screen with radius $r$ is given by $E=M c^{2}$. In other words, seven physical quantities $\left(k_{\mathrm{B}} T, c, \hbar, G, a_{0}, M, r\right)$ play a fundamental role in the description of the energy $E$ of a screen. Since there are three independent dimensions (length, time and mass), Buckingham's $\Pi$ theorem demands the energy of the screen to be given by [21]:

$$
E=\frac{1}{2} N k_{\mathrm{B}} T F\left(x, \frac{T}{T_{*}}, \frac{\lambda}{r}, \frac{l_{\mathrm{p}}}{r}\right),
$$

where $F$ is an unknown function of four dimensionless parameters, $x$ was defined in Equation (1), $T_{*}:=a_{0} \hbar / c k_{\mathrm{B}}$ is a quantity with dimensions of temperature - which coincides to the Unruh temperature evaluated at the acceleration $a_{0}$-and $\lambda:=c^{2} / a_{0}$ is a characteristic length that appears when relativistic effects are considered in the extended regime of gravity [17]. The dimensionless factor $N / 2$ has been introduced for consistency with the standard law of equipartition. It is important to note that the temperature $T_{*}$ is proportional to the so called "cut off Debye temperature" given in Equation (3) and constitutes a characteristic temperature scale in an extended regime of gravity where MONDian effects are to be taken in consideration.

The ratio $l_{\mathrm{p}} / r$ appears as a characteristic dimensionless quantity in Equation (7), but Equation (4) suggests that this quantity is closely related to the holographic principle and not to a modification to the equipartition law. Also, since that ratio does not contain $a_{0}$ it will be incapable to account for any MOND phenomenology. In other words, the equipartition energy $E$ is only a function of three dimensionless parameters: $\quad x, T / T_{*}$ and $\lambda / r$. The simplest assumption to make for the function $F$ is that it is of power-law form on any of its arguments, i.e.:

$$
E=\frac{\xi}{2} N k_{\mathrm{B}} T x^{\alpha}\left(\frac{T}{T_{*}}\right)^{\beta}\left(\frac{\lambda}{r}\right)^{\gamma},
$$

where $\xi$ is a constant of proportionality.

In the remaining of this section, we study three separate cases associated with the previous relation:

Case (A)

Let us consider $\alpha=\gamma=0$ in Equation (8) to obtain:

$$
E=\frac{\xi}{2} N k_{\mathrm{B}} T\left(\frac{T}{T_{*}}\right)^{\beta} .
$$

The physics behind this choice of parameters can be understood under the basis of the case studied by [20] since, as it was mentioned previously, the temperature $T_{*}$ corresponds-except for a $2 \pi$ factor-with the Unruh temperature on a holographic screen. In that particular study, the authors dealt with a non-standard equipartition law of energy including the one dimensional Debye function to take into account the corrections at low temperatures. Their equipartition law corresponds to (9), with $T$ representing the one dimensional Debye function.

Equating relation (9) to $M c^{2}$, and using Equation (4), the temperature can be written as:

$$
T=\frac{\hbar}{c k_{\mathrm{B}}}\left(\frac{a_{0}^{\beta} G M}{2 \pi \xi r^{2}}\right)^{1 /(\beta+1)} .
$$

Substituting this into (5), and employing (6), as made by Verlinde, the resultant entropic force is:

$$
F=2 \pi m\left(\frac{a_{0}^{\beta} G M}{2 \pi \xi_{1} r^{2}}\right)^{1 /(\beta+1)} .
$$

Taking $\beta=0$ and $\xi=1$ in Equation (9), the standard equipartition law is obtained and as seen from relation (11), Newton's gravitational law is recovered. In order to obtain a MONDian $1 / r$ gravitational force law we must take $\beta=1$ and $\xi=2 \pi$ :

$$
F=m \frac{\sqrt{a_{0} G M}}{r} .
$$


In other words, the equipartition energy must satisfy the following condition:

$$
E=\frac{1}{2} N k_{\mathrm{B}} T \begin{cases}1, & \text { for Newtonian gravity, } \\ 2 \pi T / T_{*}, & \text { for MOND-like gravity. }\end{cases}
$$

\section{Case (B)}

Let us now consider the case when $\beta=\gamma=0$ in Equation (8).

Once again, using the equivalence between mass and energy, and the explicit form of Planck's area, we can write:

$$
M c^{2}=\xi \frac{2 \pi r^{2} c^{3}}{G \hbar} k_{\mathrm{B}} T x^{\alpha},
$$

which with the aid of Equations (5) and (6), gives following expression for the entropic force:

$$
F=\frac{G m M}{\xi x^{\alpha} r^{2}} .
$$

The choice $\alpha=0$ and $\xi=1$ results into Newton's law of gravity and $\alpha=1$ with $\xi=1$ converge to MOND's force Formula (12).

The acceleration exerted on the test mass $m$ is then given by:

$$
a=\frac{a_{0}}{r^{2}} \frac{G M}{a_{0}} \frac{1}{x^{\alpha}}=a_{0} \frac{x^{2}}{x^{\alpha}} .
$$

It is convenient to express it in this form, since we can easily compare it with (1). It can be observed that given the form proposed for the energy $E$, in the Newtonian regime, the ratio $x^{2} / x^{\alpha} \rightarrow x^{2}$, and in the modified one, $x^{2} / x^{\alpha} \rightarrow x$, which are the same limits satisfied by the function $f(x)$, as discussed in Section 1 . Since the transition function $f(x)$ is given by Equation (2) it follows that the equipartition energy can be written in a very general form as:

$$
E=\frac{1}{2} N k_{\mathrm{B}} T x \frac{1 \pm x^{n}}{1 \pm x^{n+1}}
$$

\section{Case (C)}

Finally, we study the case for which $\alpha=\beta=0$ in Equation (8) is considered. Equating the resulting equation to $M c^{2}$ it is possible to find the temperature in terms of the mass and distance and so with the aid of relation (5) it follows that:

$$
F=G \frac{m M}{\xi r^{2}}\left(\frac{a_{0} r}{c^{2}}\right)^{\gamma}
$$

The choice $\gamma=0$ and $\xi=1$ yields Newton's gravitational law. A MOND-like limit $1 / r$ seems to be possible when $\gamma=1$, but the square root dependence on the mass cannot be achieved with this choice. Furthermore, the speed of light still appears on this non-relativistic limit. This all means that the dimensionless parameter $\lambda / r$ must not appear on Equation (8), which means that $\gamma=0$.

To summarise this section, note that the inclusion of Milgrom's acceleration constant as a fundamental quantity of nature related to gravitational phenomena, is capable of generalising the equipartition law in such a way that either Newton or MOND force formulae can be obtained. This result reinforces the idea that, at all scales, gravity is an emergent force with a thermodynamic nature.

\section{Modifications to the Holographic Principle}

Let us now search for a MOND-like force formula assuming that the equipartition law has its usual form and allowing for modifications related to the way in which information is stored on the holographic screens (cf. Equation (4)). The only additional constant that needs to be taken into consideration is $a_{0}$ and so, Buckingham's 
theorem of dimensional analysis requires that the number of bits $N$ stored on a screen is given by:

$$
N=\frac{4 \pi r^{2}}{l_{\mathrm{P}}^{2}} g(x),
$$

where $g(x)$ is an unknown dimensionless function. Since Equation (19) is a generalisation of the standard relation (4), the function $g(x) \rightarrow 1$ in the Newtonian regime of gravity, i.e. for $x \gg 1$. The previous equation assumes the validity of the holographic principle and only changes the quantitative way in which information is stored on a particular screen. Let us assume that the function $g(x)$ is a power law, i.e. $g(x)=x^{\zeta}$, to obtain:

$$
N=\frac{4 \pi r^{2}}{l_{\mathrm{p}}^{2}} x^{\zeta},
$$

With this in mind, we can follow Verlinde's analysis. Assuming the validity of the equipartition law of energy, and the equivalence between mass and energy inside the screen, along with Equation (20), it follows that:

$$
T=\frac{G M \hbar}{2 \pi c k_{B} r^{2} x^{\zeta}} .
$$

Direct substitution of the previous equation on relation (6), with the aid of the first law of thermodynamics (5) yields the following expression for the acceleration caused by this entropic force:

$$
a=\frac{G M}{r^{2} x^{\zeta}} .
$$

The choice $\zeta=1$ yields a MONDian gravitational force and $\zeta=0$ a Newtonian one. Comparison of the previous equation with (1) it follows that the complete transition (19) is given by:

$$
N=\left(\frac{4 \pi r^{2}}{l_{\mathrm{P}}^{2}}\right) \times \frac{1 \pm x^{n}}{1 \pm x^{n+1}} .
$$

Finally, a possible alternative way to modify Verlinde's result it to combine these two approaches, i.e. assume that both the equipartition law of energy and the holographic principle get modified when MONDian effects are introduced. Based on the previous analysis, this can be studied if we introduce the parameter $x$ as follows:

$$
E=\frac{1}{2} N k_{\mathrm{B}} T p(x), \quad N=\frac{4 \pi r^{2}}{l_{\mathrm{p}}^{2}} q(x),
$$

where $p(x)$ and $q(x)$ are unknown functions of $x$. Given that both $p$ and $q$ are proportional to $E$ and $N$ respectively, they will be inversely proportional to the gravitational acceleration, i.e.:

$$
a=\frac{G M}{r^{2}} \frac{1}{p(x) q(x)}=a_{0} \frac{x^{2}}{p(x) q(x)},
$$

and so,

$$
p(x) q(x)=\frac{x^{2}}{f(x)},
$$

according to Equation (1) with $p(x)$ and $q(x)$ tending to 1 in the Newtonian limit, i.e. when $x \gg 1$. Also, the previous equation imposes the following restriction: $p(x) g(x) \rightarrow x$ in the MONDian regime of gravity, i.e. $x \ll 1$.

\section{Discussion}

As explained by [22] [23] and in a more profound and empirical way by [24], if gravitational phenomena require to be modified at a certain scales of mass and length one needs to incorporate a new fundamental constant of nature relevant to all gravitational phenomena at those scales. This gravitational constant is as important as 
Newton's constant of gravity and can be mathematically manipulated as to have dimensions of acceleration which converge to Milgrom's acceleration constant $a_{0}$. This is so since gravitational phenomena do not follow the standard Newtonian (or general relativistic) behaviour of gravity at scales which greatly differ from the ones in which precise gravitational experiments have been performed to test the validity of Newton's law of gravitation (or Einstein's general relativity - cf. [25]). The behaviour of gravity at those scales can be considered as independent of the behaviour of standard gravity and as such a new fundamental constant of nature has to be introduced into the description of gravitational phenomena, which is a standard procedure to follow when extensions of a particular physical theory are performed (e.g. [21]).

In this article we have introduced this extra fundamental constant of nature $a_{0}$ in the description of gravity and used thermodynamic and information properties of space and time in order to show that a MONDian force law can be obtained by assuming the validity of the holographic principle. Specifically, this has been studied under two different approaches using Buckingham's $\Pi$ theorem of dimensional analysis:

1) In the first approach, the equipartition law is modified and the holographic principle keeps its standard form, resulting on a temperature scale $T_{*}$ (corresponding to the Unruh temperature evaluated at the Milgrom acceleration $a_{0}$ ). This temperature corresponds to the one already found by [20] who studied the problem of the emergence of gravity modifying the equipartition law using a Debye model. [26] also worked on modifications focused on thermodynamical statistical properties and in the same way as [20] dynamical modifications with its corresponding MOND transition function $\mu\left(a / a_{0}\right)$ were obtained. As explained by [15], direct dimensional analysis points towards a modification of the gravitational force, and the calculations we performed in this work were done by keeping Newton's second law untouched, allowing for an extension on the gravitational sector.

2) In a second approach, modifications to the holographic principle — with the equipartition law unchangedhave showed to be able to explain in a natural way how gravity transits from a Newtonian regime to a modified one.

The important point about these two different ways is that they are consistent with the general formula for acceleration experienced by a particle under a gravitational field given in (1). In this sense, and in the context of emergent gravity, it suggests that the transition observed across different astrophysical systems could be a consequence of a modification at a deeper level in the equipartition law and/or in the holographic principle, i.e. the observed effects at large scales in gravitational systems reflect the behaviour of physical laws at a deeper thermodynamical level. More generally, it has been also considered the possibility of modifications of both the holographic principle and the equipartition law in such regimes.

As pointed by [9], observations of globular clusters yield a lower limit on the exponent $n \gtrsim 8$ in Equation (2), meaning that the transition function $f(x)$ is quite abrupt, i.e. $f(x)=x$ for $x \leq 1$ (MONDian regime) and $f(x)=x^{2}$ for $x \geq 1$ (Newtonian regime), with almost no soft transition from one regime to the other. This means that the transition functions $E, N, p(x)$ and $q(x)$ calculated in this article must present a rather abrupt transition.

A full non-relativistic theory of gravity can be constructed assuming a modification of inertia as described by [22], but as shown in this work the modification naturally appears in the force sector and not on the dynamical one.

With a few natural assumptions about space and information, the main result of this article is to show that gravity can be considered an emergent phenomenon also in the MONDian regime. This suggests that the force of gravity on this extended regime is not a fundamental force of nature, but a consequence of the inherent properties of space and time. Since [1] showed that Newtonian gravity emerges from the thermodynamic properties of space and time, this all suggests that gravitation is an emergent phenomenon at all scales of mass and length.

\section{Acknowledgements}

We thank an anonymous referee for his fruitful comments on the first version of this article. This work was supported by a DGAPA-UNAM grant (PAPIIT IN111513-3) and a CONACyT grant (240512). DAC and SM thank support granted by CONACyT 480147 and 26344. The authors gratefully acknowledge the comments made by Ehoud Pazy and Hristu Culetu for the valuable comments made of an earlier version of this article.

\section{References}

[1] Verlinde, E. (2011) Journal of High Energy Physics, 4, 29. http://dx.doi.org/10.1007/JHEP04(2011)029 
[2] Bekenstein, J.D. (1973) Physical Review D, 7, 2333-2346. http://dx.doi.org/10.1103/PhysRevD.7.2333

[3] Bekenstein, J.D. (1974) Physical Review D, 9, 3292-3300. http://dx.doi.org/10.1103/PhysRevD.9.3292

[4] Bardeen, J.M., Carter, B. and Hawking, S.W. (1973) Communications in Mathematical Physics, 31, 161-170. http://dx.doi.org/10.1007/BF01645742

[5] Hawking, S.W. (1974) Nature (London), 248, 30-31. http://dx.doi.org/10.1038/248030a0

[6] Townsend, P.K. (1997) Black Holes. ArXiv General Relativity and Quantum Cosmology e-Prints.

[7] Padmanabhan, T. (2010) Reports on Progress in Physics, 73, Article ID: 046901. http://dx.doi.org/10.1088/0034-4885/73/4/046901

[8] Jacobson, T. (1995) Physical Review Letters, 75, 1260-1263. http://dx.doi.org/10.1103/PhysRevLett.75.1260

[9] Hernandez, X. and Jiménez, M.A. (2012) Astrophysical Journal, 750, 9. http://dx.doi.org/10.1088/0004-637X/750/1/9

[10] Hernandez, X., Jiménez, M.A. and Allen, C. (2012) European Physical Journal C, 72, 1884. http://dx.doi.org/10.1140/epjc/s10052-012-1884-6

[11] Mastropietro, C. and Burkert, A. (2008) Monthly Notices of the Royal Astronomical Society, 389, 967-988. http://dx.doi.org/10.1111/j.1365-2966.2008.13626.x

[12] Lee, J. and Komatsu, E. (2010) The Astrophysical Journal, 718, 60-65. http://dx.doi.org/10.1088/0004-637X/718/1/60

[13] Thompson, R. and Nagamine, K. (2012) Monthly Notices of the Royal Astronomical Society, 419, 3560-3570. http://dx.doi.org/10.1111/j.1365-2966.2011.20000.x

[14] Moffat, J.W. and Toth, V.T. (2010) Can Modified Gravity (MOG) Explain the Speeding Bullet (Cluster)? http://arxiv.org/abs/1005.2685

[15] Mendoza, S., Hernandez, X., Hidalgo, J.C. and Bernal, T. (2011) Monthly Notices of the Royal Astronomical Society, 411, 226-234. http://dx.doi.org/10.1111/j.1365-2966.2010.17685.x

[16] Milgrom, M. (1982) The Astrophysical Journal, 270, 371-389. http://dx.doi.org/10.1086/161131

[17] Bernal, T., Capozziello, S., Hidalgo, J.C. and Mendoza, S. (2011) European Physical Journal C, 71, 1794. http://dx.doi.org/10.1140/epjc/s10052-011-1794-z

[18] Carranza, D.A., Mendoza, S. and Torres, L.A. (2013) European Physical Journal C, 73, 2282. http://dx.doi.org/10.1140/epjc/s10052-013-2282-4

[19] Mendoza, S., Bernal, T., Hernandez, X., Hidalgo, J.C. and Torres, L.A. (2013) Monthly Notices of the Royal Astronomical Society, 433, 1802-1812. http://dx.doi.org/10.1093/mnras/stt752

[20] Sheykhi, A. and Sarab, K.R. (2012) Journal of Cosmology and Astroparticle Physics, 2012, 12. http://dx.doi.org/10.1088/1475-7516/2012/10/012

[21] Sedov, L.I. (1959) Similarity and Dimensional Methods in Mechanics. Academic Press, Waltham.

[22] Famaey, B. and McGaugh, S.S. (2012) Living Reviews in Relativity, 15, 10. http://dx.doi.org/10.12942/lrr-2012-10

[23] Mendoza, S. (2011) Extending Cosmology: The Metric Approach. http://arxiv.org/abs/1208.3408

[24] Mendoza, S. and Olmo, G. (2012) Living Reviews in Relativity, 15, 10.

[25] Will, C.M. (1992) Theory and Experiment in Gravitational Physics. Cambridge University Press, Cambridge.

[26] Pazy, E. and Argaman, N. (2012) Physical Review D, 85, Article ID: 104021. http://dx.doi.org/10.1103/PhysRevD.85.104021 